\title{
Seleção de cultivares de cana-de-açúcar para a Zona da Mata Norte de Pernambuco III: Final de safra
}

\section{Selection of sugarcane cultivars to the North Forest Zone of Pernambuco III: Final crop}

\author{
Aurélia Pietrina da Costa Albuquerque ${ }^{1}$, Gerson Quirino Bastos ${ }^{2}$, João de Andrade Dutra Filho ${ }^{3}$, Lauter Silva Souto ${ }^{4}$, Patricio \\ Borges Maracajá ${ }^{5}$ Djalma Euzébio Simões Neto ${ }^{6}$.
}

Resumo: Sendo os cultivares melhorados a base de sustentação e desenvolvimento do setor sucroeneregético no Brasil, objetivou-se com este trabalho avaliar o desempenho agroindustrial de 26 cultivares de cana-de-açúcar na microrregião canavieira da Mata Norte de Pernambuco. O experimento foi conduzido na área agrícola da Usina Olho D’água, onde foi utilizado o delineamento experimental em blocos casualizados com quatro repetições. As variáveis analisadas foram: toneladas de pol por hectare, toneladas de cana por hectare, fibra, pol \% corrigida, pureza, teor de sólidos solúveis e açúcar total recuperável. Realizou-se a análise de variância conjunta dos experimentos e estimativa de parâmetros genéticos, as médias foram agrupadas pelo teste de Scott e Knott ao nível de 5\% de probabilidade. Realizou-se ainda a rentabilidade econômica bruta de cada cultivar. Constatou-se que a seleção de cultivares superiores deve ser baseada nas variáveis TPH e TCH. Como opções de cultivo comercial para a terceira época de colheita destacam-se como mais produtivas, RB92579, RB867515 e RB93509.

Palavras-chave: melhoramento vegetal, genética biometrica, Saccharum spp.

\begin{abstract}
Improved cultivars are support base and development of energétic and alcohol sector in Brazil, aimed with this work was to evaluate the agroindustrial performance of 26 sugarcane cultivars in the microrregion da Mata Norte of Pernambuco. The experiment was conducted in the agricultural area of the sugarmill Olho d'gua, which was used a randomized block with four replications. The variables analyzed were tons of pol per hectare, sugarcane tons per hectare, fiber, corrected pol\%, purity, soluble solids content and total recoverable sugar. Were conducted the variance analysis and estimation of genetic parameters, the averages were grouped by the Scott and Knott test at 5\% probability. It was held still gross economic profitability of each cultivar. The selection of superior cultivars should be based on TPH and TCH variables. As commercial cultivation options for the third harvest season stand out as more productive, RB92579, RB867515 e RB93509.
\end{abstract}

Key-words: plant breeding, biometric genetic, Saccharum spp.

\footnotetext{
*Autor para correspondência

Recebido para publicação em 30/01/2016; aprovado em 12/03/2016

${ }^{1}$ Departamento de Agronomia (DEPA), Universidade Federal Rural de Pernambuco (UFRPE), 52171-900, Recife, PE, Brasil.

${ }^{2}$ Departamento de Agronomia, UFRPE, Rua Dom Manoel de Medeiros, s/n, Dois Irmãos, 52171-900 Recife (PE). E-mail: cjose@ ufrpe.br;

${ }^{3}$ Prof. D. Sc. da UAGRA - UFCG - Pombal - PB, filho-dutra@ig.com.br,luizjose@ hotmail.com

4 Professor, Centro de Ciências e Tecnologia Agroalimentar, Universidade Federal de Campina Grande; Pombal, PB, Brasil. E-mail: lautersouto@yahoo.com.br

${ }^{5}$ Professor, Programa de Pós-graduação em Sistemas Agroindustriais, Universidade Federal de Campina Grande; Pombal, PB, Brasil. Email:patriciomaracaja@gmail.com

${ }^{6}$ Universidade Federal Rural de Pernambuco (UFRPE), Estação Experimental de Cana-de-açúcar de Carpina, Rua Ângela Cristina de Luna, Bairro Novo, CEP 55810-700 Carpina, PE, Brasil. E-mail:desn@oi.com.br
} 


\section{INTRODUÇÃO}

A cana-de-açúcar assume grande importância econômica, sendo considerada uma das culturas agrícolas mais importantes em regiões de clima tropical e subtropical, pelas grandes áreas plantadas, por gerar produtos como o açúcar, o álcool, bicombustível e o bagaço, que é um resíduo da cana utilizado para produção de eletricidade (ALMEIDA et. al., 2009).

A qualidade dos materiais genéticos e os ganhos em produtividade são resultados dos conhecimentos científicos originados dos diversos programas de melhoramento genético ao longo dos anos, principalmente os trabalhos sobre estabilidade fenotípica e avaliação contínua da produtividade que permite um aumento dos ganhos genéticos, proporcionados pela distribuição otimizada dos cultivares nos vários ambientes (DUTRA FILHO et. al., 2014).

Este crescimento na produtividade se deve a muitos fatores tais como: técnicas de manejo, desenvolvimento e implantação de uma moderna tecnologia agrícola, entretanto merece maior destaque os programas de melhoramento genético através do desenvolvimento contínuo de novas variedades mais produtivas com características agronômicas favoráveis, resistentes as principais pragas e doenças (DUTRA FILHO et. al., 2011a).

Os programas de melhoramento genético de cana-deaçúcar têm como objetivo desenvolver, selecionar e recomendar cultivares mais produtivos, adaptáveis às condições de cada região de cultivo (SILVA, 2008). Para ser enquadrada como um cultivar ideal, o mesmo deve apresentar características como: elevada produtividade e baixo grau de flutuação em seu desempenho agroindustrial quando cultivado sob anos agrícolas sucessivos e diferentes épocas de colheita.

No Estado de Pernambuco, experimentos com a cultura da cana-de-açúcar são colhidos em início de safra, que corresponde aos meses de setembro e outubro; meio de safra, a novembro e dezembro; e final de safra, a janeiro e fevereiro; em três ciclos de colheita, cana planta, cana soca e cana ressoca, respectivamente. Desta forma, de posse dos dados, tem-se a possibilidade de avaliar a magnitude das interações (genótipos $\mathrm{x}$ ciclos de colheita), que geralmente são significativas (SOUZA et. al., 2012).

O objetivo do presente trabalho foi avaliar o desempenho agroindustrial de 26 cultivares de cana-de-açúcar na microrregião canavieira da Mata Norte de Pernambuco e selecionar os que se destacam para colheita em final de safra.

\section{MATERIAL E MÉTODOS}

De acordo com a caracterização edafoclimática das regiões canavieiras de Pernambuco, classificadas por Koffler et al. (1986), realizou-se a instalação e condução do experimento na área agrícola da Usina Central Olho D’água localizada na microrregião canavieira da Mata Norte de Pernambuco, no Município de Camutanga com as seguintes coordenadas geográficas $\left(07^{\circ} 24^{\prime} \mathrm{S}\right.$ e $\left.35^{\circ} 16^{\prime} \mathrm{W}\right)$, altitude de 98 $\mathrm{m}$ durante as safras agrícolas 2005/2006, 2006/2007 e 2007/2008.

O delineamento experimental utilizado foi o de blocos completos casualizados com quatro repetições. Foram avaliados 26 cultivares comerciais, conforme identificado (Tabela 1).
Tabela 1. Identificação dos genótipos de cana-de-açúcar, quanto à procedência.

\begin{tabular}{lc}
\hline Variedades & Procedência \\
\hline 1. RB867515 & RIDESA \\
2. RB92579 & RIDESA \\
3. SP81-3250 & COPERSUCAR \\
4. Q138 & AUSTRÁLIA \\
5. RB863129 & RIDESA \\
6. SP79-1011 & COPERSUCAR \\
7. RB93509 & RIDESA \\
8. RB75126 & RIDESA \\
9. RB942520 & RIDESA \\
10. SP78-4764 & COPERSUCAR \\
11. RB892700 & RIDESA \\
12. RB953180 & RIDESA \\
13. RB942898 & RIDESA \\
14. RB953281 & RIDESA \\
15. RB952900 & RIDESA \\
16. RB942991 & RIDESA \\
17. RB72454 & RIDESA \\
18. RB872552 & RIDESA \\
19. RB943365 & RIDESA \\
20. RB952675 & RIDESA \\
21. RB928064 & RIDESA \\
22. RB942849 & RIDESA \\
23. RB813804 & RIDESA \\
24. RB943161 & RIDESA \\
25. RB943066 & RIDESA \\
26. RB943538 & RIDESA \\
\hline
\end{tabular}

As parcelas experimentais foram constituídas por 5 linhas de $8 \mathrm{~m}$, com espaçamento de $1 \mathrm{~m}$ entre linhas e $1 \mathrm{~m}$ entre plantas, totalizando uma área útil de $40 \mathrm{~m}^{2}$.

As correções de $\mathrm{pH}$ do solo e adubações do campo experimental foram realizadas conforme o sistema de produção canavieira da empresa agroindustrial. Os materiais utilizados neste trabalho, clones e variedades foram oriundos de sementeira do PMGCA/UFRPE/RIDESA, conduzida na mesma região.

As variáveis analisadas foram: toneladas de pol por hectare (TPH), toneladas de cana por hectare $(\mathrm{TCH})$, fibra (FIB), pol \% corrigida (PCC), pureza (PZA), teor de sólidos solúveis (BRIX) e açúcar total recuperável (ATR).

Para estimar a produtividade por área $(\mathrm{TCH})$, foi realizada a pesagem de todos os colmos da parcela, obtendose o valor em $\mathrm{kg}$, e, posteriormente, os mesmos foram transformados em TCH através da seguinte equação (Peso total da parcela x 10 / área útil da parcela $\mathrm{em} \mathrm{m}^{2}$ ). Para obter a Tonelada de pol por hectare (TPH), foi realizado o cálculo baseado na produtividade agrícola e na pol \% corrigida (TCH x PCC / 100), obtendo-se assim a produtividade industrial.

As variáveis pureza (PZA), teor de sólidos solúveis (BRIX), açúcar total recuperável (ATR), pol \% corrigida (PCC) e fibra (FIB) foram estimadas de acordo com a metodologia proposta por Fernandes (FERNANDES, 2003).

Com o intuito de apresentar o comportamento dos genótipos em termos econômicos, foi realizada a análise da rentabilidade econômica bruta utilizando a seguinte expressão: valor em $\mathrm{kg}$ de Açúcares Totais Recuperáveis (ATR) x Tonelada de cana por hectares $(\mathrm{TCH}) \times$ Preço do $\mathrm{kg}$ de Açúcares Totais Recuperáveis (ATR). 
A análise de variância conjunta de experimentos foi realizada segundo o modelo estatístico apresentado por Cruz (2006): $\mathrm{Y}_{\mathrm{ijk}=} \mu+(\mathrm{b} / \mathrm{c})_{\mathrm{jk}}+\mathrm{g}_{\mathrm{i}}+\mathrm{c}_{\mathrm{k}}+\mathrm{gc}_{\mathrm{ik}}+\varepsilon_{\mathrm{ijk}}$

Onde:

$\mathrm{Y}_{\mathrm{ijk}}$ : observação do i-ésimo genótipo, avaliado no jésimo bloco dentro do k-ésimo corte

$\mu$ : média geral do ensaio;

$(\mathrm{b} / \mathrm{c})_{\mathrm{jk}}$ : efeito do bloco j dentro do corte k;

$\mathrm{g}_{\mathrm{i}}$ : efeito do tratamento (ou genótipo) $\mathrm{i}$;

$\mathrm{c}_{\mathrm{k}}$ : efeito do corte $\mathrm{k}$;

$\mathrm{gc}_{\mathrm{ik}}$ : efeito da interação entre o genótipo i e o corte $\mathrm{k}$ e; $\varepsilon_{\mathrm{ijk}}$ : erro aleatório associado a observação ijk.

Foram determinados como fixos, os efeitos de médias $(\mu)$ e genótipos $(\mathrm{g})$, e aleatórios os efeitos do bloco (b), corte (c), interação genótipo corte (gc) e o erro experimental $(\varepsilon)$. Os resultados da análise de variância conjunta dos três cortes dos três experimentos foram obtidos através do esquema apresentado na tabela 2 .

Para verificar a homogeneidade das variâncias residuais, antes da execução da análise de grupos de experimentos, foi realizado o teste $\mathrm{F}$ máximo de Hartley, onde, de acordo com as recomendações de Gomes (1990), todas as variáveis apresentaram um valor inferior a 7 (sete) para a razão entre a maior e a menor variância do erro.

Ainda segundo Cruz (2006), foram estimados os seguintes parâmetros:

$$
\hat{\varphi}_{\mathrm{g}}^{2}=\frac{\text { QMG }-\mathrm{QMGA}}{\mathrm{cr}}
$$

genética:

Componente de variância da interação genótipo $\mathrm{x}$ corte:

$$
\begin{aligned}
& \sigma_{\mathrm{gc}}^{2}=\frac{\mathrm{QMGC}-\mathrm{QMR}}{\mathrm{r}} \frac{\mathrm{g}-1}{\mathrm{~g}} \\
& \text { Herdabilidade média: } \mathrm{h}^{2}=\frac{\hat{\varphi}_{\mathrm{g}}^{2}}{(\mathrm{QMG} / \mathrm{cr})}
\end{aligned}
$$

$$
\begin{aligned}
& \text { Coeficiente } \\
& \mathrm{CV}_{\mathrm{g}}=\frac{\left(100 \sqrt{\hat{\varphi}_{\mathrm{g}}^{2}}\right)}{\mathrm{m}}
\end{aligned}
$$

Índice $b: \quad \mathrm{CV}_{\mathrm{g}} / \mathrm{CV}_{\mathrm{e}}=\sqrt{\frac{\hat{\varphi}_{\mathrm{g}}^{2}}{\sigma^{2}}}$

As médias foram agrupadas pelo teste de Scott e Knott a 5\% de probabilidade, as análises genético-estatísticas foram processadas com o auxílio do programa Genes (CRUZ, 2006).

\section{RESULTADOS E DISCUSSÕES}

Foram detectadas, através do teste $\mathrm{F}$, diferenças significativas a $1 \%$ de probabilidade $(\mathrm{P}<0,01)$, para os caracteres toneladas de pol por hectare (TPH), toneladas de cana por hectare $(\mathrm{TCH})$ e teor de sólidos solúveis (BRIX) e a $5 \%$ para (PCC) e (ATR). Indicando a ocorrência de variabilidade genética entre os cultivares (Tabela 2).

Para os ciclos de colheita considerados (cana planta, soca e ressoca), observam-se diferenças significativas a $1 \%$ de probabilidade em relação as variáveis toneladas de pol por hectare (TPH), toneladas de cana por hectare $(\mathrm{TCH})$, fibra (FIB), pol \% corrigida (PCC), pureza (PZA) e açúcares totais recuperáveis $(\mathrm{ATR})$ e a $5 \%$ de probabilidade $(\mathrm{P}<0,05)$ para teor de sólidos solúveis (BRIX). De acordo com Rosse et al. (2002), os fatores edafoclimáticos nos diferentes ciclos de colheita se comportam como ambientes contrastantes (Tabela 2).

Em relação a interação genótipo $\mathrm{x}$ corte (ciclos de colheita), houve significância, para a maioria dos caracteres considerados, com exceção do caráter pureza (PZA) indicando desempenho agronômico estável dos clones e das variedades comerciais em relação a este caráter nos diferentes ciclos de colheita para o final da safra na região canavieira onde foi conduzido o experimento. Melo et al., (2009) encontraram resultados semelhantes para essas variáveis ao avaliarem o desempenho agroindustrial de cultivares de canade-açúcar no Litoral Sul de Pernambuco. Segundo esses autores a análise da interação genótipo x ciclo de colheita

\begin{tabular}{|c|c|c|c|c|c|c|c|c|}
\hline \multirow[b]{2}{*}{ F.V } & \multirow[b]{2}{*}{ G.L } & \multicolumn{7}{|c|}{ Quadrados médios } \\
\hline & & TPH & ТСH & FIB & PCC & PZA & BRIX & ATR \\
\hline Genótipos & 25 & $16,41 * *$ & $552,04 * *$ & $2,3^{\mathrm{ns}}$ & $3,61 *$ & $14,84^{\mathrm{ns}}$ & $4,92 * *$ & $297,31 *$ \\
\hline Corte & 2 & $955,86 * *$ & $4397,54 * *$ & $111,82 * *$ & $51,86 * *$ & $262,67 * *$ & $20,11 *$ & $5723,98 * *$ \\
\hline $\mathbf{G} \times \mathbf{C}$ & 50 & $5,66 * *$ & $221,18^{* *}$ & $1,64 *$ & $1,77 *$ & $16,94^{\mathrm{ns}}$ & $2,17 *$ & $143,26 *$ \\
\hline Resíduo & 225 & 2,49 & 38,57 & 0,89 & 1,24 & 16,06 & 1,52 & 94,52 \\
\hline Médias & & 11,21 & 78,58 & 14,46 & 14,22 & 86,6 & 20,22 & 140,8 \\
\hline C.V $(\%)$ & & 14,06 & 11,98 & 6,53 & 7,84 & 4,63 & 6,1 & 6,9 \\
\hline$>\mathbf{Q M R} /$ <QMR & & 2,4 & 2,86 & 3,19 & 2,63 & 10,85 & 1,93 & 2,22 \\
\hline
\end{tabular}
sinaliza ao melhorista a longevidade dos cultivares (Tabela2).

Tabela 2. Resumo da análise de variância conjunta avaliada em grupos de experimentos conduzidos na fase de competição de variedades na segunda terceira de colheita (final de safra) na Microrregião canavieira da Mata Norte de Pernambuco, Usina Central Olho D’água, Camutanga - PE, anos agrícolas 2005/2006, 2006/2007 e 2007/2008.

**e * Significativo a 1 e $5 \%$ de probabilidade respectivamente, pelo teste F; ns, não significativo, pelo teste F; G x C Interação Genótipo x Corte

Observando os parâmetros estimados (tabela 3), constata-se como na primeira e segunda época de colheita o valor do índice b inferior a uma unidade, variância da interação genótipo $\mathrm{x}$ corte superior ao componente de variância genética, para os caracteres toneladas de cana por hectare (TCH), fibra (FIB) e pureza (PZA), podendo-se concluir que as variedades não apresentam, para esta época de colheita, elevado potencial para fins de melhoramento. Entretanto, é possível selecionar materiais superiores para toneladas de pol por hectare (TPH) e toneladas de cana por 
hectare (TCH) entre os que foram avaliados no presente valor da determinação genotípica em nível de média trabalho para serem cultivados e colhidos no final da safra apresentou média magnitude para TPH, TCH, PCC, BRIX e nesta microrregião canavieira, sempre tendo em vista que o ATR.

Tabela 3. Parâmetros estimados em três cortes das características avaliadas na fase de competição de variedades na terceira época de colheita (final de safra) em experimento conduzidos na Microrregião canavieira da Mata Norte de Pernambuco, Usina Central Olho D’água, Camutanga - PE, anos agrícolas 2005/2006, 2006/2007 e 2007/2008.

\begin{tabular}{cccccc}
\hline & \multicolumn{5}{c}{ Parâmetros } \\
\hline Caracteres & $\phi_{\mathrm{g}}^{2}$ & $\phi_{\mathrm{gc}}^{2}$ & $\mathrm{~h}^{2}$ & $\mathrm{CV}_{\mathrm{g}}$ & $\mathrm{CV}_{\mathrm{g}} / \mathrm{CV}_{\mathrm{c}}$ \\
\hline TPH & 0,90 & 0,76 & 65,50 & 8,44 & 0,60 \\
TCH & 27,57 & 31,88 & 59,93 & 6,68 & 0,56 \\
FIB & 0,05 & 0,18 & 28,59 & 1,61 & 0,25 \\
PCC & 0,15 & 0,13 & 50,89 & 2,75 & 0,35 \\
PZA & 0 & 0,21 & 0 & - & 0,39 \\
BRIX & 0,23 & 0,16 & 55,97 & 2,37 & 0,37 \\
ATR & 12,84 & 11,71 & 51,82 & 2,54 & Herdabilidade \\
$\phi 2 \mathrm{~g}:$ média; Comp: Coeficiente de
\end{tabular}

variação genético; CVg/CVc: Índice b

Pelo teste de Skott \& Knott aplicado (Tabela 4), a 5\% de probabilidade $(\mathrm{P}<0,05)$, constata-se que, para o caráter toneladas de pol por hectare (TPH), houve formação de grupos superiores onde no grupo "a" enquadram-se os genótipos RB92579, RB867515, RB93509, SP81-3250, RB75126, RB943365, RB942991, RB892700, SP79-1011, RB953281, SP78-4764 e RB928064.

Para o caráter toneladas de cana por hectare $(\mathrm{TCH})$, houve formação de grupos superiores, onde no grupo "a"

enquadram-se os genótipos RB92579, RB867515, RB93509, SP81-3250, RB75126, RB943365, RB942991, RB892700, SP79-1011, RB953281, SP78-476, RB928064 e RB863129.

Em relação às outras variáveis analisadas os clones e as variedades comerciais não formaram grupos distintos. tabela 5 .

A rentabilidade econômica bruta está explicitada na

Tabela 4. Valores médios dos caracteres TPH, TCH, FIB,PCC, PZA,BRIX e ATR para a $3^{\text {a }}$ época de colheita, final de safra, avaliados na fase de competição de variedades considerando os cultivos de cana planta, soca e ressoca (3 cortes) em grupos de experimentos conduzidos na região na Microrregião canavieira da Mata Norte de Pernambuco, Usina Central Olho D’água, Camutanga - PE, anos agrícolas 2005/2006, 2006/2007 e 2007/2008.

\begin{tabular}{|c|c|c|c|c|c|c|c|c|c|c|c|c|c|c|}
\hline & \multicolumn{14}{|c|}{ Variáveis } \\
\hline Variedades & TPH(t/ha) & & $\mathrm{TCH}(\mathrm{t} / \mathrm{ha})$ & & $\mathrm{FIB}(\%)$ & & $\mathrm{PCC}(\%)$ & & PZA(\%) & & BRIX (\%) & & $\operatorname{ATR}(\mathrm{kg} / \mathrm{t})$ & \\
\hline RB92579 & 13,84 & $\mathrm{a}$ & 94,17 & $\mathrm{a}$ & 13,53 & $\mathrm{a}$ & 14,78 & A & 87,53 & $\mathrm{a}$ & 20,48 & $\mathrm{a}$ & 145,49 & $\mathrm{a}$ \\
\hline RB867515 & 13,46 & $\mathrm{a}$ & 90,92 & $\mathrm{a}$ & 14,26 & $\mathrm{a}$ & 14,81 & A & 85,76 & $\mathrm{a}$ & 21,22 & $\mathrm{a}$ & 146,55 & $\mathrm{a}$ \\
\hline RB93509 & 12,56 & $\mathrm{a}$ & 84,83 & $\mathrm{a}$ & 14,49 & $\mathrm{a}$ & 14,95 & A & 86,28 & $\mathrm{a}$ & 21,31 & $\mathrm{a}$ & 147,49 & $\mathrm{a}$ \\
\hline SP81-3250 & 12,46 & $\mathrm{a}$ & 86,25 & $\mathrm{a}$ & 14,46 & $\mathrm{a}$ & 14,40 & A & 86,32 & $\mathrm{a}$ & 20,55 & $\mathrm{a}$ & 142,55 & $\mathrm{a}$ \\
\hline RB75126 & 12,29 & $\mathrm{a}$ & 87,17 & $\mathrm{a}$ & 14,11 & $\mathrm{a}$ & 13,96 & A & 86,13 & $\mathrm{a}$ & 19,76 & $\mathrm{a}$ & 139,09 & $\mathrm{a}$ \\
\hline RB943365 & 11,98 & $\mathrm{a}$ & 80,92 & $\mathrm{a}$ & 14,33 & $\mathrm{a}$ & 14,61 & A & 87,66 & $\mathrm{a}$ & 20,47 & $\mathrm{a}$ & 144,81 & $\mathrm{a}$ \\
\hline RB942991* & 11,92 & $\mathrm{a}$ & 80,00 & $\mathrm{a}$ & 13,67 & $\mathrm{a}$ & 14,83 & A & 86,95 & $\mathrm{a}$ & 20,74 & $\mathrm{a}$ & 145,80 & $\mathrm{a}$ \\
\hline RB892700 & 11,83 & $\mathrm{a}$ & 82,67 & $\mathrm{a}$ & 14,93 & $\mathrm{a}$ & 14,33 & A & 87,06 & $\mathrm{a}$ & 20,45 & $\mathrm{a}$ & 140,87 & $\mathrm{a}$ \\
\hline SP79-1011 & 11,64 & $\mathrm{a}$ & 79,25 & $\mathrm{a}$ & 14,97 & $\mathrm{a}$ & 14,70 & A & 87,45 & $\mathrm{a}$ & 20,92 & $\mathrm{a}$ & 144,41 & $\mathrm{a}$ \\
\hline RB953281* & 11,56 & $\mathrm{a}$ & 84,25 & $\mathrm{a}$ & 14,54 & $\mathrm{a}$ & 13,61 & A & 85,93 & $\mathrm{a}$ & 19,53 & $\mathrm{a}$ & 135,46 & a \\
\hline SB78-4764 & 11,45 & $\mathrm{a}$ & 80,00 & $\mathrm{a}$ & 14,68 & $\mathrm{a}$ & 14,23 & A & 87,21 & $\mathrm{a}$ & 20,19 & $\mathrm{a}$ & 140,16 & $\mathrm{a}$ \\
\hline RB928064 & 11,41 & $\mathrm{a}$ & 81,92 & $\mathrm{a}$ & 15,13 & $\mathrm{a}$ & 13,89 & A & 87,64 & $\mathrm{a}$ & 19,79 & $\mathrm{a}$ & 136,45 & $\mathrm{a}$ \\
\hline RB72454 & 11,15 & $\mathrm{~b}$ & 75,58 & $\mathrm{~b}$ & 13,62 & $\mathrm{a}$ & 14,76 & A & 86,46 & $\mathrm{a}$ & 20,73 & $\mathrm{a}$ & 145,96 & $\mathrm{a}$ \\
\hline RB863129 & 11,11 & b & 79,92 & $\mathrm{a}$ & 14,25 & $\mathrm{a}$ & 13,92 & A & 86,55 & a & 19,75 & $\mathrm{a}$ & 138,08 & a \\
\hline RB942898* & 10,94 & $\mathrm{~b}$ & 78,00 & $\mathrm{~b}$ & 14,57 & $\mathrm{a}$ & 13,95 & A & 86,47 & $\mathrm{a}$ & 19,84 & $\mathrm{a}$ & 138,19 & $\mathrm{a}$ \\
\hline RB813804 & 10,90 & $\mathrm{~b}$ & 73,92 & $\mathrm{~b}$ & 14,71 & $\mathrm{a}$ & 14,73 & A & 87,96 & $\mathrm{a}$ & 20,72 & $\mathrm{a}$ & 145,17 & $\mathrm{a}$ \\
\hline RB942520* & 10,76 & b & 70,25 & b & 14,93 & $\mathrm{a}$ & 15,15 & A & 87,88 & $\mathrm{a}$ & 21,42 & $\mathrm{a}$ & 150,03 & $\mathrm{a}$ \\
\hline RB872552 & 10,72 & b & 73,58 & b & 14,65 & $\mathrm{a}$ & 14,7 & A & 87,6 & $\mathrm{a}$ & 20,74 & $\mathrm{a}$ & 144,59 & $\mathrm{a}$ \\
\hline Q138 & 10,64 & b & 78,00 & b & 14,21 & $\mathrm{a}$ & 13,8 & A & 85,90 & $\mathrm{a}$ & 19,67 & $\mathrm{a}$ & 136,86 & $\mathrm{a}$ \\
\hline RB943161* & 10,63 & $\mathrm{~b}$ & 74,00 & $\mathrm{~b}$ & 14,64 & $\mathrm{a}$ & 14,28 & $\mathrm{~A}$ & 87,74 & $\mathrm{a}$ & 20,12 & $\mathrm{a}$ & 141,16 & $\mathrm{a}$ \\
\hline RB953180* & 10,57 & $\mathrm{~b}$ & 74,83 & b & 14,06 & $\mathrm{a}$ & 14,01 & A & 87,01 & $\mathrm{a}$ & 19,7 & $\mathrm{a}$ & 139,17 & $\mathrm{a}$ \\
\hline RB952900* & 10,30 & b & 74,67 & b & 14,43 & $\mathrm{a}$ & 13,86 & A & 85,94 & $\mathrm{a}$ & 19,85 & $\mathrm{a}$ & 137,08 & $\mathrm{a}$ \\
\hline RB942849* & 9,45 & b & 69,33 & b & 14,18 & $\mathrm{a}$ & 13,46 & A & 82,59 & $\mathrm{a}$ & 19,93 & $\mathrm{a}$ & 136,14 & $\mathrm{a}$ \\
\hline RB943538* & 9,43 & b & 71,08 & b & 15,01 & $\mathrm{a}$ & 13,10 & A & 86,20 & $\mathrm{a}$ & 18,93 & $\mathrm{a}$ & 129,70 & a \\
\hline RB952675* & 9,38 & $\mathrm{~b}$ & 69,83 & $\mathrm{~b}$ & 14,43 & $\mathrm{a}$ & 13,42 & A & 85,67 & $\mathrm{a}$ & 19,3 & $\mathrm{a}$ & 133,56 & $\mathrm{a}$ \\
\hline RB943066* & 9,30 & $\mathrm{~b}$ & 67,75 & $\mathrm{~b}$ & 15,12 & $\mathrm{a}$ & 13,62 & A & 85,79 & $\mathrm{a}$ & 19,7 & $\mathrm{a}$ & 136,03 & $\mathrm{a}$ \\
\hline
\end{tabular}

Médias seguidas da mesma letra pertencem ao mesmo grupo pelo teste de Scott \&knott, ao nível de 5\% de probabilidade. 
Tabela 5. Valores econômicos, em reais por hectare, dos 11 clones e 15 variedades comerciais avaliadas na fase de competição de variedades na segunda época de colheita (meio de safra) considerando os cultivos de cana planta, soca e ressoca (3 cortes) em grupos de experimentos conduzidos na região canavieira da Mata Norte de Pernambuco usina Olho Central D'água, Camutanga-PE, anos agrícolas 2005/2006, 2006/2007 e 2007/2008.

\begin{tabular}{|c|c|}
\hline Variedades & Valor econômico baseado em ATR t/ha \\
\hline RB92579 & $\mathrm{R} \$ 6.850,40$ \\
\hline RB867515 & $\mathrm{R} \$ 6.662,16$ \\
\hline RB93509 & $\mathrm{R} \$ 6.255,79$ \\
\hline SP813250 & $\mathrm{R} \$ 6.147,47$ \\
\hline RB75126 & $\mathrm{R} \$ 6.062,24$ \\
\hline RB943365 & $\mathrm{R} \$ 5.859,01$ \\
\hline RB942991 & $\mathrm{R} \$ 5.832,00$ \\
\hline RB892700 & $\mathrm{R} \$ 5.822,86$ \\
\hline SP791011 & $\mathrm{R} \$ 5.722,25$ \\
\hline RB953281 & $\mathrm{R} \$ 5.706,25$ \\
\hline SB784764 & $\mathrm{R} \$ 5.606,40$ \\
\hline RB928064 & $\mathrm{R} \$ 5.588,99$ \\
\hline RB863129 & $\mathrm{R} \$ 5.517,68$ \\
\hline RB72454 & $\mathrm{R} \$ 5.515,83$ \\
\hline RB942898 & $\mathrm{R} \$ 5.389,41$ \\
\hline RB813804 & $\mathrm{R} \$ 5.365,48$ \\
\hline Q138 & $\mathrm{R} \$ 5.337,54$ \\
\hline RB872552 & $\mathrm{R} \$ 5.319,47$ \\
\hline RB942520 & $\mathrm{R} \$ 5.269,80$ \\
\hline RB943161 & $\mathrm{R} \$ 5.222,92$ \\
\hline RB953180 & $\mathrm{R} \$ 5.207,05$ \\
\hline RB952900 & $\mathrm{R} \$ 5.117,88$ \\
\hline RB942849 & $\mathrm{R} \$ 4.719,29$ \\
\hline RB952675 & $\mathrm{R} \$ 4.663,25$ \\
\hline RB943538 & $\mathrm{R} \$ 4.609,54$ \\
\hline RB943066 & $\mathrm{R} \$ 4.608,02$ \\
\hline
\end{tabular}

Constata-se como às três melhores opções de cultivo para colheita no final da safra os genótipos RB92579, RB867515 e RB93509, apresentando receita da ordem de R\$ $6.850,40, \mathrm{R} \$ 6.662,16$ e $\mathrm{R} \$ 6.255,79$ por quantidade de ATR por hectare respectivamente.

\section{CONCLUSÕES}

A seleção de cultivares pode ser realizada com base nas variáveis TCH e TPH.

Os cultivares RB92579, RB867515 e RB93509 são os mais produtivos e opções de cultivo para colheita no final da safra.

\section{REFERÊNCIAS BIBLIOGRÁFICAS}

ALMEIDA, C.M.A.; LIMA, S.E.N.; LIMA, G.S.A.; BRITO, J.Z.; DONATO, V.M.T.S.; SILVA, M.V. Caracterização molecular de cultivares de cana-de-açúcar utilizando marcadores ISSR. Ciência e Agrotecnologia, v. 33, p. 17711776, 2009.

CRUZ, C.D. 2006. Programa GENES: aplicativo computacional em genética e estatística. Editora UFV, Viçosa, Brasil. 2006. 442p.
DUTRA FILHO, J.A.; BASTOS, G.Q.; MACHADO, P.R.; SILVA, L.J.; SIMÕES NETO, D.E.; CHAVES, A.; SILVA, F.S.G. Estimativa do ganho de seleção para a produtividade em famílias de cana-de-açúcar. Comunicata Scientiae, v. 3, n. 1, p. 35-40.

DUTRA FILHO, J.A., MELO, L.J.O.T., RESENDE, L.V., ANUNCIAÇÃO FILHO, C.J., BASTOS, G.Q. Aplicação de técnicas multivariadas no estudo da divergência genética em cana-de-açúcar. Revista Ciência Agronômica, v. 42, n. 1, p. 185-192, 2011b.

FERNANDES, A.C. Cálculos na agroindústria da cana-deaçúcar. 2.ed. Piracicaba: EME, 2003. 240p.

GOMES, F. P. Curso de estatística experimental.13. ed. Piracicaba: USP, 1990. 467 p.

KOFFLER, N.F., LIMA, J.F.W.F., LACERDA, M.F., SANTANA, J.F., SILVA, M.A. Caracterização edafoclimática das regiões canavieiras do Brasil: PERNAMBUCO. Editora IAA, Piracicaba, Brasil. 1986. 78p.

DUTRA FILHO, J.A.; CALSA JUNIOR, T.; SIMÕES NETO, D.E. 2014. Phenotype adaptability and stability of sugarcane genotypes in the sugarcane belt of the State of Pernambuco, Brazil. Genetics and Molecular Research, v. 13, n. 3 , p. $6865-6877,2014$

MELO, L.J.O.T.; OLIVEIRA, F.J.; BASTOS, G.Q.; ANUNCIAÇÃO FILHO, C.J.; REIS, O.V. 2009. Desempenho agroindustrial de variedades de cana-de- açúcar na zona da mata litoral sul de Pernambuco. Ciência e Agrotecnologia, v. 33, n. 3, p. 684-691, 2009.

ROSSE, L.N.; VENCOVSKY, R.; FERREIRA, D.F. Comparação de métodos de regressão para avaliar a estabilidade fenotípica em cana-de-açúcar. Pesquisa Agropecuária Brasileira, Brasília, v. 37, n. 1, p. 25-32, 2002.

SILVA, M. A. Interação genótipo x ambiente e estabilidade fenotípica de cana-de-açúcar em ciclo de cana de ano. Bragantia, v. 67, n. 1, p. 109-117, 2008.

SOUZA, P.H.N.; BASTOS, G.Q.; ANUNCIAÇÃO FILHO, C.J.; DUTRA FILHO, J.A.; MACHADO, P.R. 2012. Avaliação de genótipos de cana-de-açúcar para inicio de safra na Microrregião Centro de Pernambuco. Revista Ceres, v. 59, n. 5 : p. $427-432,2012$. 